\title{
Paracrine factors from mesenchymal stem cells attenuate epithelial injury and lung fibrosis
}

\author{
QINQIN SHEN $^{1}$, BING CHEN $^{2}$, ZHIFENG XIAO $^{2}$, LIFEN ZHAO $^{1}$, XUEFENG XU $^{1}$, \\ XUAN WAN $^{1}$, MULAN JIN ${ }^{3}$, JIANWU DAI ${ }^{2}$ and HUAPING DAI ${ }^{1}$ \\ ${ }^{1}$ Beijing Key Laboratory of Respiratory and Pulmonary Circulation, Beijing Institute of Respiratory Medicine, \\ Department of Respiratory and Critical Care Medicine, Beijing Chao-Yang Hospital, \\ Capital Medical University, Beijing 100020; ${ }^{2}$ Key Laboratory of Molecular Development Biology, \\ Institute of Genetics and Developmental Biology, Chinese Academy of Sciences, Beijing 100190; \\ ${ }^{3}$ Department of Pathology Medicine, Beijing Chao-Yang Hospital, \\ Capital Medical University, Beijing 100020, P.R. China
}

Received November 20, 2013; Accepted June 26, 2014

DOI: $10.3892 / \mathrm{mmr} .2014 .3092$

\begin{abstract}
Paracrine factors are currently considered to be the major mechanism through which mesenchymal stem cells (MSCs) exert their actions. The aim of this study was to investigate the protective effects of conditioned medium (CM) from bone marrow mesenchymal stem cells (MSC) on bleomycin (BLM)-induced lung injury and fibrosis, both in vitro and in vivo. A549 human non-small cell lung cancer epithelial cells were cultured in serum-free medium, or MSC-CM, both with or without BLM. The protective effects of MSC-CM was determined by MTT assay to assess cell viability and Annexin V-PE to assess apoptosis. Rats were intratracheally injected with MSC-CM, saline, or conditioned medium from fibroblasts on day 0 and day 3 after intratracheal administration of BLM, and were sacrificed on day 28. Lung injury and fibrosis were assessed by histological assessment, Ashcroft score, and hydroxyproline assay; lung cell apoptosis was detected using terminal deoxynucleotidyl transferase dUTP nick end labeling assay. In comparison to the control group $(0.17 \pm 0.01), 8$ and $16 \%$ MSC-CM had a significant stimulatory effect on A549 cellular proliferation $(0.24 \pm 0.03$ and $0.24 \pm 0.04$,
\end{abstract}

Correspondence to: Dr Huaping Dai, Beijing Key Laboratory of Respiratory and Pulmonary Circulation, Beijing Institute of Respiratory Medicine, Department of Respiratory and Critical Care Medicine, Beijing Chao-Yang Hospital, Capital Medical University, 8 Gongren Tiyuchang South Road, Beijing 100020, P.R. China E-mail: daihuaping@ccmu.edu.cn

Professor Jianwu Dai, Key Laboratory of Molecular Development Biology, Institute of Genetics and Developmental Biology, Chinese Academy of Sciences, 3 Nanyitiao, Zhongguancun, Beijing 100190, P.R. China

E-mail: jwdai@genetics.ac.cn

Key words: mesenchymal stem cells, lung fibrosis, conditioned medium, apoptosis, paracrine respectively, $\mathrm{P}<0.01)$. A549 cells cultured with $\mathrm{MSC}-\mathrm{CM}$ were protected from BLM-induced apoptosis, 23.43 $\pm 3.76 \%$ vs. 38.06 $\pm 4.32 \%$; $(\mathrm{P}<0.05)$. In the BLM-challenged rats, MSC-CM was shown to protect against lung fibrosis in terms of lung inflammation, fibrotic scores, collagen deposition, and cell apoptosis. This data suggests that MSCs are capable of protecting against lung injury and fibrosis both in vitro and in vivo through a paracrine anti-inflammatory mechanism. MSC-CM may provide a novel approach for the treatment of lung fibrosis.

\section{Introduction}

Idiopathic pulmonary fibrosis (IPF) is a chronic progressive fibrotic lung disease of unknown origin with a median survival time of $<3$ years $(1,2)$. To date, only lung transplantation has been proved to be effective in the treatment of IPF, due to its poorly understood etiology and pathogenesis $(1,2)$. Increasing evidence has demonstrated that recurrent or continuous injury to the lung epithelium initiates and promotes lung fibrosis $(3,4)$. Unresolved injury can lead to compromised proliferative capacity of the alveolar epithelial cells (AECs), increased cell death (either necrosis or apoptosis), and a phenotypic switch of the surviving cells $(5,6)$. Apoptosis of AECs has been observed in lung biopsies of IPF patients $(7,8)$ as well as in BLM-challenged experimental models of lung fibrosis (9) in areas adjacent to fibroblast foci. Transplantation of alveolar epithelial cells, aimed at protecting cells from endogenous and exogenous injuries and stimulating regeneration, has been shown to ameliorate BLM-induced lung fibrosis (10). In addition, hepatocyte growth factor (HGF) and keratinocyte growth factor (KGF), which are both capable of promoting development and proliferation of AECs, can attenuate BLM-induced lung fibrosis (11-13). Therefore, any treatment capable of promoting proliferation or reducing apoptosis of AECs may ameliorate pulmonary fibrosis.

Bone marrow mesenchymal stem cells (BM-MSCs) are a useful tool in cellular therapy of injured tissues. BM-MSCs can 
differentiate into mature non-hematopoietic cells of multiple tissues that can facilitate the repair of injured organs, such as the liver, lung, heart and kidney (14). However, numerous studies have shown that lung epithelial or endothelial cells are rarely derived from BM-MSCs $(15,16)$, therefore engraftment in the lung as structural epithelium or endothelium is not currently considered the mechanism by which BM-MSCs can repair lung tissue (17). Previous evidence has shown however, that BM-MSCs can migrate to injured tissues, communicate with injured parenchyma cells, and function in the wound healing process through the production of paracrine-soluble cytokines and growth factors, which modulate the regeneration of the epithelium and endothelium (18).

Previous research has revealed that systemic infusion of conditioned medium (CM) obtained from mesenchymal stem cells (MSCs) leads to a hepato-protective response in the acutely injured liver, specifically by inhibition of cell apoptosis and stimulation of reparative programs (19). $\mathrm{Xu}$ et al (20) showed that BM-MSCs in the lung suppressed the endotoxin-induced increase in circulating proinflammatory cytokines by paracrine factors. Additionally, MSC-CM has been shown to prevent $\mathrm{O}_{2}$-induced alveolar epithelial cell apoptosis, accelerate alveolar epithelial cell wound healing, and enhance endothelial cord formation (21). The aim of the present study was to investigate the protective effects of MSC-CM on BLM-induced alveolar epithelial injury in vitro, and pulmonary fibrosis in vivo.

\section{Materials and methods}

Isolation and culture of BM-MSCs. Isolation and expansion of MSCs was performed as described previously (22). Briefly, BM-MSCs were obtained from 4 week-old male Sprague Dawley rats by flushing the femur and tibia cavities with phosphate-buffered saline (PBS). Following isolation, the cell suspension was centrifuged, and the cells were plated in Dulbecco's modified Eagle's medium (DMEM) (Gibco-BRL, Grand Island, NY, USA) supplemented with $10 \%$ fetal bovine serum (FBS; Gibco-BRL), 2 mm L-glutamine (Gibco-BRL), $0.1 \mathrm{mmol} / \mathrm{l}$ non-essential amino acids (HyClone, Logan, UT, USA), $100 \mathrm{IU} / \mathrm{ml}$ penicillin (Gibco-BRL) and $100 \mathrm{IU} / \mathrm{ml}$ streptomycin (Gibco-BRL) in culture dishes (Corning-Costar, Corning, NY, USA). Non-adherent cells were removed after $30 \mathrm{~h}$ of incubation, and BM-MSCs were recovered by their tight adhesion to the culture dishes. BM-MSCs were identified phenotypically by their typical fibroblast-like appearance and by detection of immune cell surface markers specific for CD45, CD34, CD11b, CD44 and CD29 (Lifespan BioSciences, USA) by flow cytometry. BM-MSCs were routinely cultured and used at passages 3 and 4 for subsequent experiments. The use of rats in the present study was approved by the Ethics Committee of the Beijing Chao-Yang Hospital (Capital Medical University, Beijing, China).

Preparation of MSC-CM. Once BM-MSCs had reached $80 \%$ confluence in $10 \mathrm{~cm}$ diameter culture dishes $\left(\sim 2 \times 10^{6}\right.$ cells $)$, the cells were washed thoroughly three times with PBS, following which they were incubated in $5 \mathrm{ml}$ serum-free DMEM for $24 \mathrm{~h}$. The CM, containing secreted proteins, was collected and filtered using a $0.45-\mu \mathrm{m}$ low molecular weight protein binding membrane. The $\mathrm{CM}$ was further concentrated 25-fold by ultrafiltration (Millipore, Bedford, MA, USA) with a 3-kDa molecular weight cut-off (19). The concentrated CM was filtered through a $0.22-\mu \mathrm{m}$ membrane, for sterilization, and stored at $-80^{\circ} \mathrm{C}$ prior to use. The CM of 3T3 fibroblasts (FB-CM) was prepared as a control and using the same methodology as described for the production of MSC-CM.

Cell proliferation assay. A549 human non-small cell lung cancer epithelial cells (ATCC, Rockefeller, MD, USA) were seeded into 48-well plates (10,000 cells/well), and were incubated with serum-free RPMI-1640 medium (Gibco-BRL) and serum-free RPMI-1640 supplemented with MSC-CM (MSC-CM/RPMI-1640), separately. Different proportions of the MSC-CM was added to the medium: 1, 2, 4, 8 and $16 \%$. After incubation for $48 \mathrm{~h}$, the cell growth-promoting activity of the MSC-CM was detected by MTT assay (Sigma, St. Louis, MO, USA). The absorbance was read at a wavelength of $492 \mathrm{~nm}$.

Apoptosis analysis by flow cytometry. A549 cells were cultured in RPMI-1640 medium supplemented with $10 \% \mathrm{FBS}$, at $37^{\circ} \mathrm{C}$ in a humidified incubator with an atmosphere of $5 \% \mathrm{CO}_{2} / 95 \%$ air. Following adherence of the cells to the culture dish, the cultures were washed thoroughly with PBS three times. A549 cells were cultured in either serum-free RPMI-1640 (BLM group) or serum-free RPMI-1640 supplemented with $8 \%$ MSC-CM (MSC-CM group) for $24 \mathrm{~h}$. BLM $(300 \mu \mathrm{g} / \mathrm{ml})$ (Nippon Kayaku Co., Tokyo, Japan) was added to the cultures, which were then incubated for a further 24 h. A549 cells cultured in serum-free RPMI-1640 for $48 \mathrm{~h}$ were used as a control group. A total of $1 \times 10^{6}$ cells were prepared from each sample for the apoptosis assay using Annexin V-PE Apoptosis Detection kit (BioVision, Mountain View, CA, USA) using an LSR flow cytometer (Becton-Dickinson, Mountain View, CA, USA) and CellQuest ${ }^{\mathrm{TM}}$ software (Becton-Dickinson). The experiments were repeated independently three times.

Pulmonary fibrosis in vivo studies. All animals used in the present study were maintained in compliance with the guidlines of the Chinese Ministry of Public Health. Twenty female Wistar rats, weighing between 200 and 220 g, were used for the pulmonary fibrosis experiment. The rats were randomly divided into four groups $(n=5)$; a control, BLM, MSC-CM, and an FB-CM group. The rats were anaesthetized by intraperitoneal injection of pentobarbital sodium at a dosage of $30 \mathrm{mg} / \mathrm{kg}$ body weight. The rats of the BLM, MSC-CM, and FB-CM groups were intratracheally administered $0.2 \mathrm{ml}$ saline, $0.2 \mathrm{ml}$ MSC-CM, and $0.2 \mathrm{ml} \mathrm{FB-CM}$, respectively, at $6 \mathrm{~h}$ and on day 3 following the intratracheal administration of BLM $(5 \mathrm{mg} / \mathrm{kg}$ body weight). The rats of the control group were given the same volume of saline. All of the rats were sacrificed on day 28 and the lung tissues were harvested. The experimental protocols were approved by the Committee on the Ethics of Animal Experiments, of Capital Medical University (Beijing, China).

Histological assessment. For histological analysis, the right lower lung lobes were fixed in $10 \%$ neutral formaldehyde for $24 \mathrm{~h}$ and embedded in paraffin. Sections of 5- $\mu \mathrm{m}$ thickness were cut and stained with hematoxylin and eosin (H\&E) and 

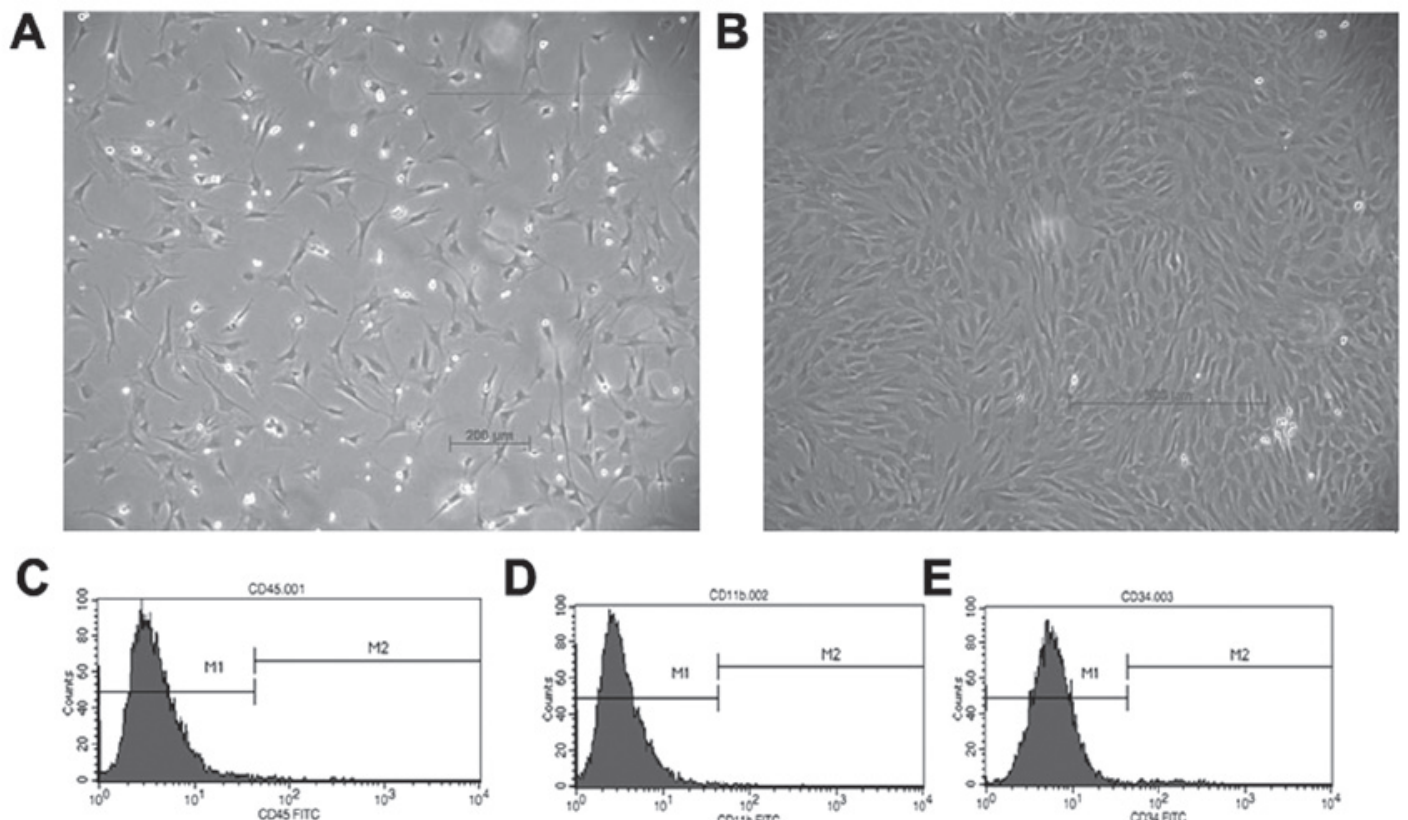

$\operatorname{CD} 45(-)$

CD11b (-)

CD34 (-)

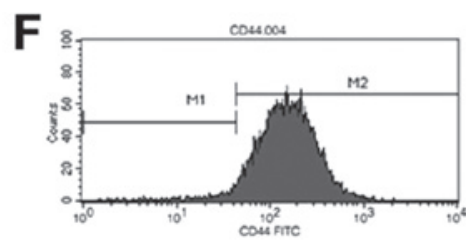

CD44 (+)
$\mathbf{G}$

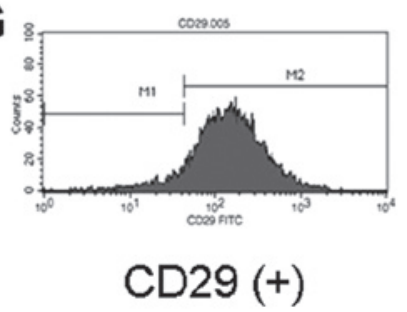

Figure 1. Phenotypical characteristics of mesenchymal stem cells in vitro. (A) Morphology of MSCs on day 12 (magnification, x200); (B) Morphology of passage 3 of MSCs (magnification, x200). (C-G) The expression of (C) CD45, (D) CD11b, (E) CD34, (F) CD44 and (G) CD29 were evaluated by flow cytometry. The MSCs were negative for CD45, CD11b, CD34 and positive for CD44 and CD29. MSCs, mesenchymal stem cells; CD, cluster of differentiation.

Masson's trichrome, separately. Histopathological evaluation of pulmonary fibrosis was performed according to the Ashcroft scoring under a photomicroscope (23).

Hydroxyproline measurement. Following sacrifice of the rats, the right upper lung lobes were rapidly frozen in liquid nitrogen and stored at $-80^{\circ} \mathrm{C}$ prior to use. The hydroxyproline content was measured by a colorimetric-based spectrophotometric assay using a Hydroxyproline Detection kit (Nanjing Jiancheng Biotechnology Company, Nanjing, China), according to the manufacturer's instructions.

Terminal deoxynucleotidyl transferase-mediated dUTP nick end labeling assays (TUNEL). Apoptotic scores were obtained by TUNEL assay using an In Situ Cell Death Detection kit (Roche-Diagnostics, Mannheim, Germany).

Statistical analysis. All of the experiments were repeated three times. Statistical analysis was performed using SPSS version 15.0 (SPSS Inc., Chicago, IL, USA). The data are expressed as the means \pm standard deviation and was statistically analyzed using an analysis of variance. Significant differences $(\mathrm{P}<0.05)$ between groups were assessed using a post hoc analysis. For comparison of pathologic grades, the Mann-Whitney U-test was used. A $\mathrm{P}<0.05$ was considered to indicate a statistically significant difference.

\section{Results}

Effects of MSC-CM on A549 cells. BM-MSCs were phenotypically identified by their typical fibroblast-like appearance (Fig. 1A and B), and by absent expression of the immune markers for CD45, CD34 and CD11b and positive expression for CD44 and CD29 on the cell surface, as determined by flow cytometry (Fig. 1C-G). In order to investigate the proliferative effect of MSC-CM, A549 cells were treated with serum-free RPMI-1640 containing different proportions of MSC-CM: $0,1,2,4,8$ and $16 \%$, for $48 \mathrm{~h}$. Results from the MTT cell viability assay showed that as compared with the control group $(0.17 \pm 0.01)$, the treatment of A459 cells with medium containing 1, 2, and 4\% MSC-CM appeared to have an effect on cell proliferation, however the differences were not statistically significant $(0.19 \pm 0.01,0.20 \pm 0.02$ and $0.20 \pm 0.03$, respectively) ( $\mathrm{P}>0.05$ ) (Fig. 2). When A549 cells were treated with medium supplemented with 8 and $16 \%$ MSC-CM, the absorption values were $0.24 \pm 0.03$ and $0.24 \pm 0.04$ respectively, which was significantly greater as compared with the control group $(\mathrm{P}<0.01)$. This indicated that $\mathrm{MSC}-\mathrm{CM}$ had a stimulatory effect on A549 cell proliferation, and the effect was correlated with the concentration of MSC-CM in the culture medium.

Inhibition of MSC-CM on A549 cell apoptosis in vitro. To determine the inhibitory effect of MSC-CM on A549 cell 
apoptosis, $8 \%$ MSC-CM was used on BLM-challenged cells. The apoptotic rate of A549 cells of the BLM group $(38.06 \pm 4.32 \%)$ was significantly increased as compared with that of the control group $(9.93 \pm 1.20 \%)(\mathrm{P}<0.01)$ (Fig. 3). Following treatment of the A549 BLM-challenged cells with $8 \%$ MSC-CM for $48 \mathrm{~h}$, the cell apoptotic rate was reduced to $(23.43 \pm 3.76 \%)$, this reduction, in comparison to the apoptotic rate of the BLM group, was statistically significant $(\mathrm{P}<0.05)$ (Fig. 3). These results indicated that MSC-CM could partially rescue A549 cells from BLM-induced apoptosis.

Anti-fibrotic effects of MSC-CM in BLM-induced lung fibrosis. The protective effects of MSC-CM were examined using a BLM-challenged rat model of lung fibrosis. As compared with the control group (Fig. 4A and E), the inflammation and collagen accumulation in the lung tissues was significantly increased in the BLM group $(\mathrm{P}<0.05$; Fig. $4 \mathrm{~B}$ and $\mathrm{F})$, and this increase was attenuated by treatment of the rats with MSC-CM (Fig. 4C and G). The Ashcroft fibrosis score was significantly increased in the BLM group as compared with the control group $(5.0 \pm 0.55$ vs. $0.8 \pm 0.20, \mathrm{P}<0.01$, Fig. $4 \mathrm{I})$. As compared with the BLM group, MSC-CM treatment significantly reduced the fibrotic scores $(3.00 \pm 0.31, \mathrm{P}<0.05$, Fig. $4 \mathrm{I})$, whereas no difference was observed following FB-CM treatment $(3.20 \pm 0.49, \mathrm{P}>0.05$, Fig. 4I).

The collagen content of the rat lung tissue was assessed by a hydroxyproline assay. The collagen production of lung tissues was significantly increased in the BLM group $(0.77 \pm 0.05 \mu \mathrm{g} / \mathrm{mg}$ lung weight), as compared with the control group $(0.65 \pm 0.09 \mu \mathrm{g} / \mathrm{mg}$ lung weight) $(\mathrm{P}<0.05$, Fig. $4 \mathrm{~J})$. In comparison to the BLM group, the hydroxyproline content of lung tissues in the MSC-CM group was significantly decreased $(0.67 \pm 0.07 \mu \mathrm{g} / \mathrm{mg}$ lung weight) $(\mathrm{P}<0.05$, Fig. $4 \mathrm{~J})$, whereas FB-CM treatment did not attenuate the collagen content in rat lung tissues $(0.78 \pm 0.03 \mu \mathrm{g} / \mathrm{mg}$ lung weight) $(\mathrm{P}>0.05)$ (Fig. $4 \mathrm{~J})$.

MSC-CM attenuates the apoptosis rate of AECs in vivo. As shown in Fig. 5, the proportion of apoptotic AECs in the BLM group $(20.14 \pm 1.61 \%)$ was significantly higher as compared with the control group $(10.64 \pm 4.48 \%)(\mathrm{P}<0.01)$. As compared with the BLM group, MSC-CM treatment had a significant protective effect on the apoptotic rate of AECs $(13.92 \pm 1.47 \%)$ $(\mathrm{P}<0.01)$, whereas there was no effect on the apoptotic rate of AECs following FB-CM treatment $(18.17 \pm 2.18 \%)(\mathrm{P}>0.05)$.

\section{Discussion}

IPF is a progressive parenchymal lung disease with a poor prognosis, as there is thus far no effective treatment. Unresolved injury-induced impaired proliferative capacity and an increased apoptotic rate of AECs represents potentially important mechanisms in the development of lung fibrosis $(4,5)$. Failed epithelium repair is followed by abnormal wound healing processes, including epithelial and fibroblast activation, cytokine production, coagulation pathway activation, neoangiogenesis, re-epithelialization and fibrosis $(4,5)$. It is therefore important to develop a method by which to abrogate AEC injury and to protect AECs from further apoptosis. The present study investigated whether MSC-CM had a therapeutic effect on BLM-induced lung epithelial injuries

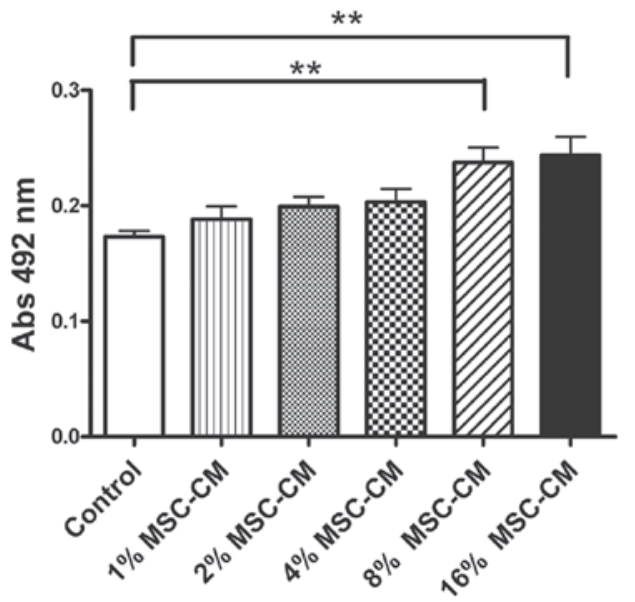

Figure 2. Effects of MSC-CM on cell proliferation. MSC-CM significantly promoted A549 human non-small cell lung cancer cell proliferation. Following treatment with serum-free RPMI-1640 supplemented with 1, 2, 4, 8 and $16 \%$ MSC-CM for $48 \mathrm{~h}$, the A549 cell growth-promoting activity was detected by MTT assay. As compared with the control, $8 \%$ and 16\% MSC-CM showed a significant effect on cell proliferation, as assessed by measuring the absorption values at $492 \mathrm{~nm}$. Data are shown as the means \pm standard error. $* * \mathrm{P}<0.01, \mathrm{n}=6$. MSC-CM, mesenchymal stem cell-conditioned media; $\mathrm{nm}$, nanometers.

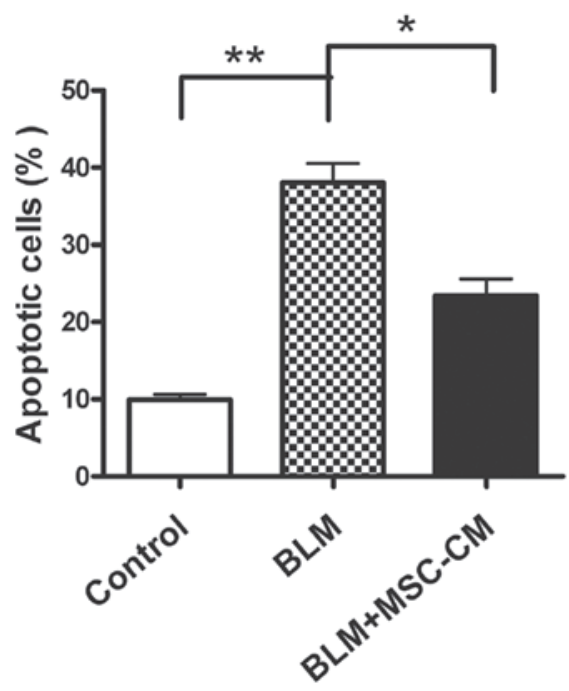

Figure 3. Effects of MSC-CM on apoptosis. MSC-CM rescued A549 human non-small cell lung cancer cell apoptosis induced by BLM. Annexin V-PE was used to detect the apoptotic rate. For each sample, $1 \times 10^{6}$ cells were prepared for the apoptosis assay using Annexin V-PE Apoptosis Detection kit using an LSR flow cytometer and CellQuest software. The apoptotic rate of A549 cells of BLM group was significantly increased as compared with the control group. Following treatment with $8 \%$ MSC-CM, the A549 cell apoptotic rate was decreased. Data are shown as the means \pm standard deviation. ${ }^{*} \mathrm{P}<0.05,{ }^{* *} \mathrm{P}<0.01, \mathrm{n}=6$. BLM, bleomycin; MSC-CM, mesenchymal stem cell-conditioned medium.

in vitro, and on pulmonary fibrosis in vivo. The major findings of the study were: (a) MSC-CM demonstrated the ability to improve viability and proliferation of A549 cells. (b) MSC-CM inhibited A549 cell apoptosis induced by BLM in serum-free conditions. (c) MSC-CM reduced the hydroxyproline content and fibrotic score of rat lung tissues treated with BLM, for which this protective effect was shown to be associated with the anti-apoptotic effects of MSC-CM. The present study 

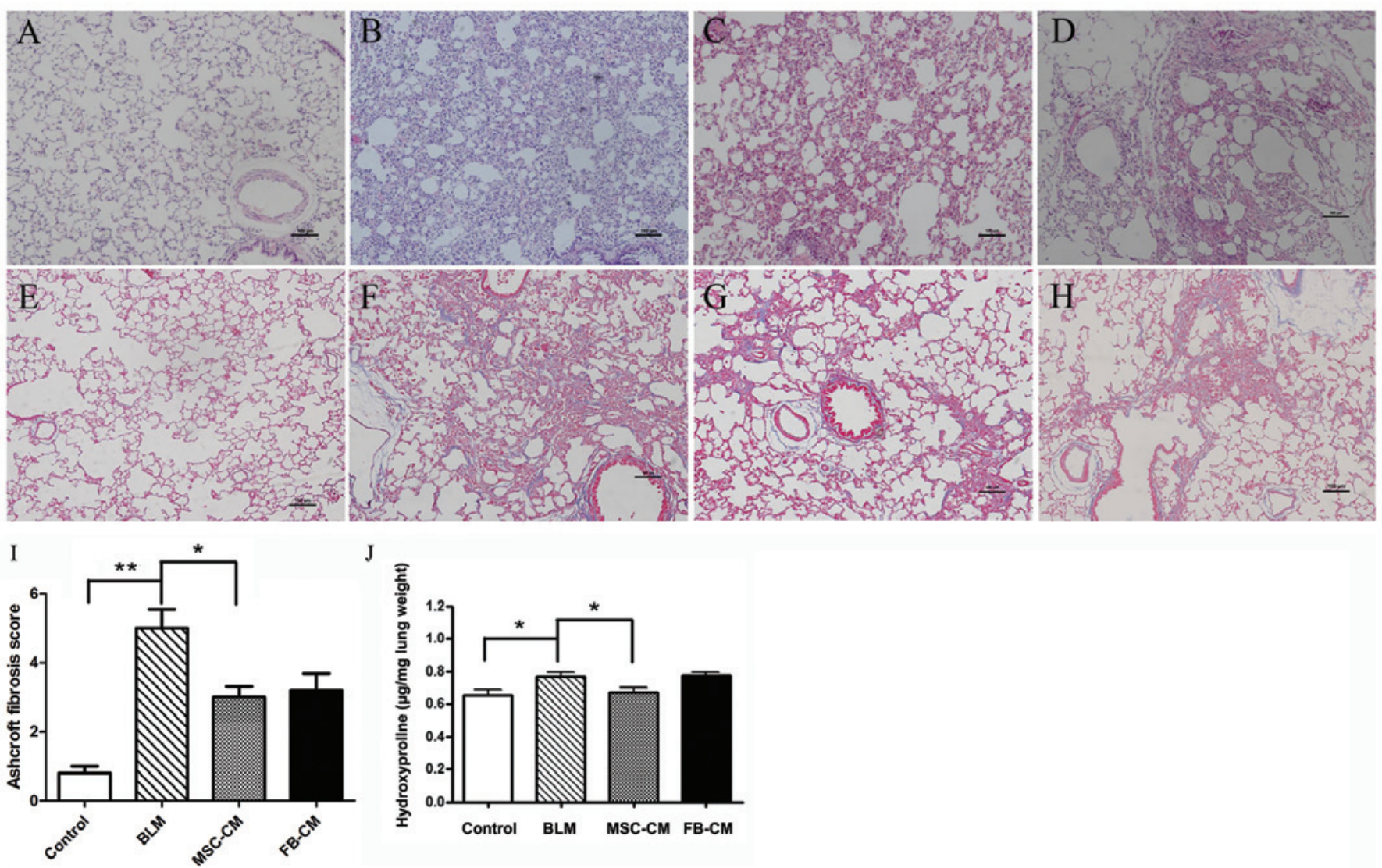

Figure 4. Effects of MSC-CM on lung fibrosis. MSC-CM attenuated BLM-induced pulmonary injury. Lung sections were stained with (A-D) hematoxylin and eosin and (E-H) Masson's trichrome, and examined under a photomicroscope. (A and E) No marked pathological changes were detected in the control group. (B and F) Thickened alveolar septa with increased infiltration of inflammatory cells and collagen deposition were observed in the BLM group. (C and G) Attenuated interstitial inflammation and collagen deposition was observed in the MSC-CM group as compared with the BLM group. (D and H) In the FB-CM group, interstitial inflammation and collagen deposition were not relieved. Scale bar $=100 \mu \mathrm{m}$; magnification, $\mathrm{x} 100$. (I) Ashcroft scoring. As compared with the BLM group, MSC-CM administration attenuated fibrotic lesions. Data are shown as the means \pm standard deviation. ${ }^{*} \mathrm{P}<0.05,{ }^{* * *} \mathrm{P}<0.01$, $\mathrm{n}=5$. (J) The collagen content was assessed by hydroxyproline assay. As compared with control rats, BLM increased collagen deposition. As compared with the BLM group, MSC-CM administration decreased the hydroxyproline content in the lung tissues, whereas FB-CM treatment did not attenuate the collagen content. Data are shown as the means \pm standard deviation. ${ }^{*} \mathrm{P}<0.05, \mathrm{n}=5$. BLM, bleomycin; MSC-CM, mesenchymal stem cell-conditioned media; FB-CM, fibroblast-conditioned media.
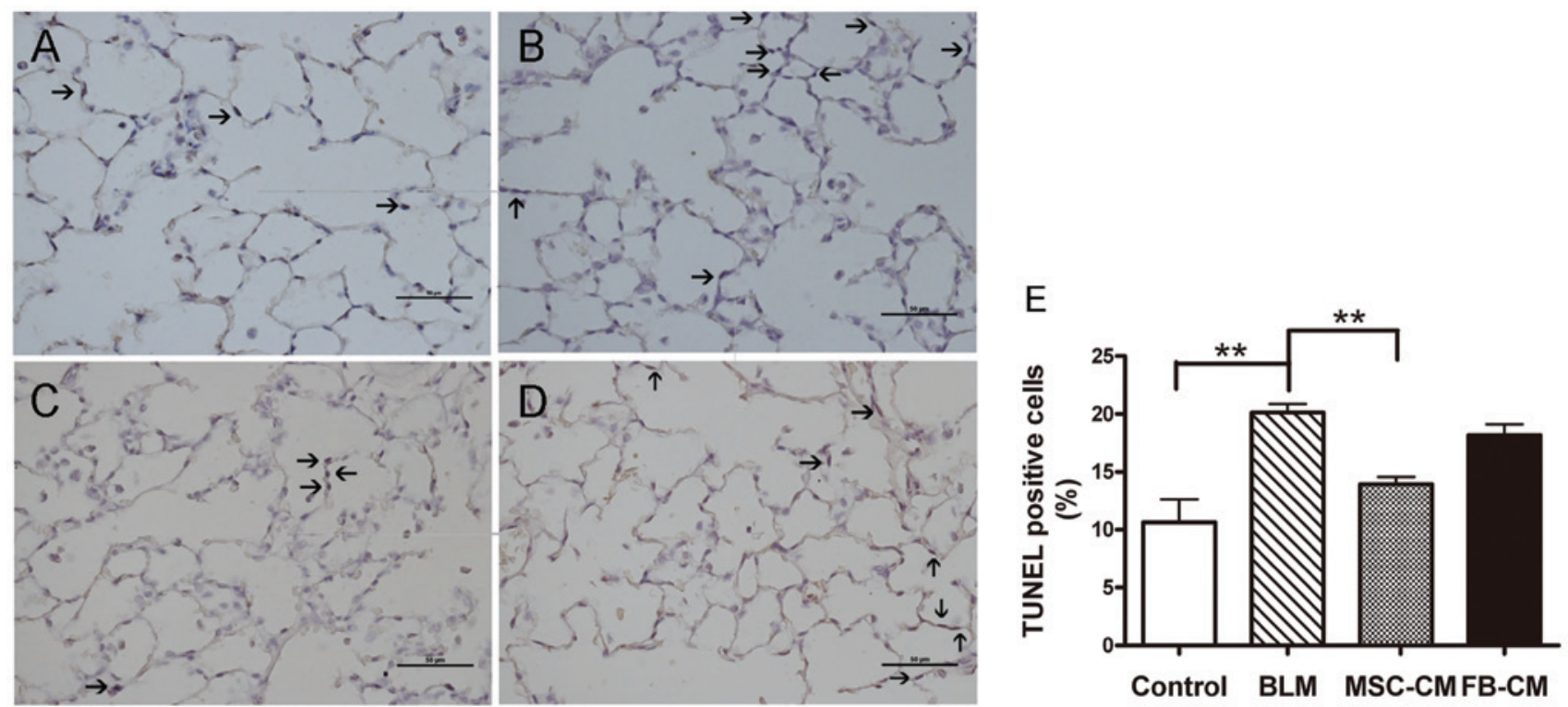

Figure 5. Analysis of apoptosis in A459 lung cells by TUNEL assay. TUNEL positive cells are indicated with an arrow. (A) In comparison with the control group, the proportion of TUNEL positive cells was significantly increased in the (B) BLM group. A lower proportion of TUNEL-positive cells were detected in the (C) MSC-CM group, as compared with the BLM group, whereas (D) FB-CM did not exhibit a protective effect. Scale bar $=50 \mu \mathrm{m}$; magnification, $\mathrm{x} 400$. Data are presented as the means \pm standard deviation. ${ }^{* * *} \mathrm{P}<0.01, \mathrm{n}=5$. BLM, bleomycin; MSC-CM, mesenchymal stem cell-conditioned media; FB-CM, fibroblast-conditioned media; TUNEL, terminal deoxynucleotidyl transferase-mediated dUTP nick-end labeling. 
provides evidence that MSC-CM has the potential to reduce apoptosis of AECs, promote AEC regeneration and inhibit development of lung fibrosis.

MSCs were first identified in the bone marrow in 1976 (24) and have since been shown to express a broad spectrum of secreted molecules, including interferon (IFN)- $\gamma$, interleukin (IL)-1 $\beta$, IL-6, IL-10, transforming growth factor (TGF)- $\beta 1$, vascular endothelial growth factor (VEGF), stromal derived factor (SDF)-1, HGF, KGF, prostaglandin PGE2, amongst others $(17,18)$. Through the action of these soluble mediators, BM-MSCs have been shown to modulate the activation, proliferation, and downstream effects of inflammatory and immune cells in both the innate and adaptive immune systems; including neutrophils, macrophages and lymphocytes $(25,26)$. However, it is unknown whether BM-MSCs are capable, through paracrine properties, of modulating AEC proliferation and apoptosis. The present study used MSC-CM, containing paracrine factors secreted from BM-MSCs, to determine the effects of BM-MSCs on lung epithelial cells. It was observed that MSC-CM enhanced the proliferation of A549 cells, and this effect was correlated with the concentration of MSC-CM (Fig. 2). MSC-CM was also shown to have an anti-apoptotic effect on A549 cells induced by BLM in vitro (Fig. 3). These findings indicate that the beneficial effects of BM-MSCs on AECs can be exerted by their paracrine properties. Previous studies have shown that the paracrine factors of MSCs have anti-apoptotic effects in vitro on cardiac cells $(27,28)$, pancreatic $\beta$ cells (29) and hepatocytes (19). BM-MSCs are known to produce several epithelial specific growth factors, including HGF and $\operatorname{KGF}(17,18)$, which have been proven to initiate a variety of biological responses including migration, proliferation, and morphogenesis in AECs (30-33). Therefore it can be speculated that the protective roles of MSC-CM on AECs may be associated with the epithelial specific growth factors secreted by BM-MSCs and the synergistic effects of these factors.

Ineffective AEC repair and increased cell apoptosis can lead to aberrant fibroblastic responses and end-stage fibrosis (5). The present in vitro study observed that BM-MSCs promoted A549 proliferation and protected against cell apoptosis induced by a BLM challenge. It has therefore been hypothesized that MSC-CM may attenuate lung fibrosis in vivo by the rescue of injured AECs. In a BLM challenged rat model of lung fibrosis, it was demonstrated that lung inflammation (Fig. 4A-D), collagen accumulation (Fig. 4E-H), hydroxyproline content (Fig. 4J) and the fibrotic score of the rat lung tissues (Fig. 4I) could be significantly decreased by MSC-CM treatment, whereas FB-CM treatment had little effect. A possible mechanism explaining the MSC-CM induced reduction in lung cell apoptosis (Fig. 5) is the generation and secretion of soluble paracrine cytokines; including HGF, KGF, PGE2, VEGF (30-33) It may also be speculated that apoptosis of AECs is mediated by reactive oxygen species, which are involved in BLM-induced lung injury and IPF $(34,35)$. It has been shown that MSCs have antioxidative activity and have a neuroprotective role in experimental autoimmune encephalomyelitis (36); therefore MSCs may inhibit the apoptosis of AECs and pulmonary fibrosis by an antioxidant effect.

The in vivo study showed that BM-MSCs attenuated lung fibrosis by paracrine properties, which has been previously demonstrated by other studies (37-39). In a BLM-induced lung fibrosis model, systemic MSC administration significantly reduced lung inflammation and damage, collagen deposition, and other injury and fibrosis markers, while only a small number of MSCs appeared to have structurally engrafted in the lung (37). Similarly, Kumamoto et al (38) and Lee et al (39) demonstrated that systemically administered BM-derived MSCs in a BLM model of lung fibrosis resulted in an improved lung injury score and modulation of inflammatory cytokine production, by protective paracrine effects. MSCs of origins other than bone marrow have also been shown to attenuate lung fibrosis. Moodley et al (40) reported that intratracheal and systemic infusion of umbilical cord derived MSCs (UC-MSCs) in a BLM model of lung injury decreased lung collagen accumulation, fibrosis score and matrix metalloproteinase expression. Based on the findings of the present study, together with previous studies, it can be concluded that administration of both MSC-CM (paracrine factors) and MSCs can attenuate lung fibrosis. The paracrine factors that mediate the protective effects of MSCs requires further study.

To our knowledge, the present study was the first to use MSC-CM, in comparison to MSCs alone, to show that MSCs are effective in reducing apoptosis of AECs induced by BLM, and enhancing AECs regeneration, resulting in fewer lung injuries, lower fibrosis scores, and reduced collagen production in the lung by their significant paracrine capacity. These effects were independent of the structural engraftment to the airway or alveolar epithelium and phenotypic switch to structural lung cells. MSC-CM may provide a novel approach for the treatment of lung fibrosis.

\section{Acknowledgements}

The present study was supported by grants from the National Natural Science Foundation of China (no. 30871120), the Sci-Tech Development Program of Beijing Municipal Education Communication (no. KZ201110025028) and the National Basic Research Program of China 973 Program (no. 2009CB522106).

\section{References}

1. King TE, Jr., Pardo A and Selman M: Idiopathic pulmonary fibrosis. Lancet 378: 1949-1961, 2011.

2. Raghu G, Collard HR, Egan JJ, et al; ATS/ERS/JRS/ALAT Committee on Idiopathic Pulmonary Fibrosis: An official ATS/ERS/JRS/ALAT statement: idiopathic pulmonary fibrosis: evidence-based guidelines for diagnosis and management. Am J Respir Crit Care Med 183: 788-824, 2011.

3. Kropski JA, Lawson WE, Young LR and Blackwell TS: Genetic studies provide clues on the pathogenesis of idiopathic pulmonary fibrosis. Dis Model Mech 6: 9-17, 2013.

4. Zoz DF, Lawson WE and Blackwell TS: Idiopathic pulmonary fibrosis: a disorder of epithelial cell dysfunction. Am J Med Sci 341: 435-438, 2011.

5. Hardie WD, Hagood JS, Dave V, et al: Signaling pathways in the epithelial origins of pulmonary fibrosis. Cell Cycle 9: 2769-2776, 2010.

6. Sakai N and Tager AM: Fibrosis of two: Epithelial cell-fibroblast interactions in pulmonary fibrosis. Biochim Biophys Acta 1832: 911-921, 2013.

7. Maher TM, Evans IC, Bottoms SE, et al: Diminished prostaglandin E2 contributes to the apoptosis paradox in idiopathic pulmonary fibrosis. Am J Respir Crit Care Med 182: 73-82, 2010.

8. Li X, Shu R, Filippatos G and Uhal BD: Apoptosis in lung injury and remodeling. J Appl Physiol (1985) 97: 1535-1542, 2004. 
9. Degryse AL, Tanjore H, Xu XC, et al: Repetitive intratracheal bleomycin models several features of idiopathic pulmonary fibrosis. Am J Physiol Lung Cell Mol Physiol 299: L442-452, 2010.

10. Serrano-Mollar A, Nacher M, Gay-Jordi G, et al: Intratracheal transplantation of alveolar type II cells reverses bleomycin-induced lung fibrosis. Am J Respir Crit Care Med 176: 1261-1268, 2007.

11. Bao S, Wang Y, Sweeney P, et al: Keratinocyte growth factor induces Akt kinase activity and inhibits Fas-mediated apoptosis in A549 lung epithelial cells. Am J Physiol Lung Cell Mol Physiol 288: L36-L42, 2005.

12. Crestani B, Marchand-Adam S, Quesnel C, et al: Hepatocyte growth factor and lung fibrosis. Proc Am Thorac Soc 9: 158-163, 2012.

13. Umeda Y, Marui T, Matsuno Y, et al: Skeletal muscle targeting in vivo electroporation-mediated HGF gene therapy of bleomycin-induced pulmonary fibrosis in mice. Lab Invest 84 836-844, 2004.

14. Krause DS, Theise ND, Collector MI, et al: Multi-organ, multi-lineage engraftment by a single bone marrow-derived stem cell. Cell 105: 369-377, 2001.

15. Rejman J, Colombo C and Conese M: Engraftment of bone marrow-derived stem cells to the lung in a model of acute respiratory infection by Pseudomonas aeruginosa. Mol Ther 17: 1257-1265, 2009.

16. Fritzell JA Jr, Mao Q, Gundavarapu S, et al: Fate and effects of adult bone marrow cells in lungs of normoxic and hyperoxic newborn mice. Am J Respir Cell Mol Biol 40: 575-587, 2009.

17. Tzouvelekis A, Ntolios P and Bouros D: Stem cell treatment for chronic lung diseases. Respiration 85: 179-192, 2013.

18. Conese M, Carbone A, Castellani S and Di Gioia S: Paracrine effects and heterogeneity of marrow-derived stem/progenitor cells: relevance for the treatment of respiratory diseases. Cells Tissues Organs 197: 445-473, 2013.

19. van Poll D, Parekkadan B, Cho CH, et al: Mesenchymal stem cell-derived molecules directly modulate hepatocellular death and regeneration in vitro and in vivo. Hepatology 47: 1634-1643, 2008

20. Xu J, Woods CR, Mora AL, et al: Prevention of endotoxin-induced systemic response by bone marrow-derived mesenchymal stem cells in mice. Am J Physiol Lung Cell Mol Physiol 293: L131-L141, 2007.

21. van Haaften T, Byrne R, Bonnet S, et al: Airway delivery of mesenchymal stem cells prevents arrested alveolar growth in neonatal lung injury in rats. Am J Respir Crit Care Med 180: $1131-1142,2009$

22. Tögel F, Weiss K, Yang Y, et al: Vasculotropic, paracrine actions of infused mesenchymal stem cells are important to the recovery from acute kidney injury. Am J Physiol Renal Physiol 292: F1626-F1635, 2007.

23. Ashcroft T, Simpson JM and Timbrell V: Simple method of estimating severity of pulmonary fibrosis on a numerical scale. J Clin Pathol 41: 467-470, 1988.

24. Phinney DG: Building a consensus regarding the nature and origin of mesenchymal stem cells. J Cell Biochem Suppl 38: 7-12, 2002.
25. Nauta AJ and Fibbe WE: Immunomodulatory properties of mesenchymal stromal cells. Blood 110: 3499-3506, 2007.

26. Lau AN, Goodwin M, Kim CF and Weiss DJ: Stem cells and regenerative medicine in lung biology and diseases. Mol Ther 20: 1116-1130, 2012.

27. Sadat S, Gehmert S, Song YH, et al: The cardioprotective effect of mesenchymal stem cells is mediated by IGF-I and VEGF. Biochem Biophys Res Commun 363: 674-679, 2007.

28. Nakanishi C, Yamagishi M, Yamahara K, et al: Activation of cardiac progenitor cells through paracrine effects of mesenchymal stem cells. Biochem Biophys Res Commun 374: 11-16, 2008.

29. Xu YX, Chen L, Wang R, et al: Mesenchymal stem cell therapy for diabetes through paracrine mechanisms. Med Hypotheses 71: 390-393, 2008.

30. Gazdhar A, Susuri N, Hostettler K, et al: HGF expressing stem cells in usual interstitial pneumonia originate from the bone marrow and are antifibrotic. PLoS One 8: e65453, 2013.

31. Gazdhar A, Temuri A, Knudsen L, et al: Targeted gene transfer of hepatocyte growth factor to alveolar type II epithelial cells reduces lung fibrosis in rats. Hum Gene Ther 24: 105-116, 2013.

32. Chakraborty S, Chopra P, Hak A, Dastidar SG and Ray A: Hepatocyte growth factor is an attractive target for the treatment of pulmonary fibrosis. Expert Opin Investig Drugs 22: 499-515, 2013.

33. Sakamoto S, Yazawa T, Baba Y, et al: Keratinocyte growth factor gene transduction ameliorates pulmonary fibrosis induced by bleomycin in mice. Am J Respir Cell Mol Biol 45: 489-497, 2011

34. Inghilleri S, Morbini P, Oggionni T, Barni S and Fenoglio C: In situ assessment of oxidant and nitrogenic stress in bleomycin pulmonary fibrosis. Histochem Cell Biol 125: 661-669, 2006.

35. Teixeira KC, Soares FS, Rocha LG, et al: Attenuation of bleomycin-induced lung injury and oxidative stress by $\mathrm{N}$-acetylcysteine plus deferoxamine. Pulm Pharmacol Ther 21: 309-316, 2008

36. Lanza C, Morando S, Voci A, et al: Neuroprotective mesenchymal stem cells are endowed with a potent antioxidant effect in vivo. J Neurochem 110: 1674-1684, 2009.

37. Ortiz LA, Gambelli F, McBride C, et al: Mesenchymal stem cell engraftment in lung is enhanced in response to bleomycin exposure and ameliorates its fibrotic effects. Proc Natl Acad Sci USA 100: 8407-8411, 2003.

38. Kumamoto M, Nishiwaki T, Matsuo N, Kimura $\mathrm{H}$ and Matsushima K: Minimally cultured bone marrow mesenchymal stem cells ameliorate fibrotic lung injury. Eur Respir J 34: 740-748, 2009.

39. Lee SH, Jang AS, Kim YE, et al: Modulation of cytokine and nitric oxide by mesenchymal stem cell transfer in lung injury/fibrosis. Respir Res 11: 16, 2010.

40. Moodley Y, Atienza D, Manuelpillai U, et al: Human umbilical cord mesenchymal stem cells reduce fibrosis of bleomycin-induced lung injury. Am J Pathol 175: 303-313, 2009. 